\title{
Intramolecular cascade radical cyclizations promoted by samarium diiodide
}

\author{
Zhang Hu, ${ }^{\text {a* }}$ Si-dong Li, ${ }^{\text {a }}$ and Peng-zhi Hong ${ }^{b}$ \\ ${ }^{a}$ Department of Chemistry, College of Science, Guangdong Ocean University, \\ Zhanjiang 524088, China \\ ${ }^{b}$ College of Food Science and Technology, Guangdong Ocean University, \\ Zhanjiang 524088, China \\ E-mail: huzhangqyx@126.com
}

DOI: $\underline{\text { http://dx.doi.org/10.3998/ark.5550190.0011.916 }}$

\begin{abstract}
Samarium diiodide promotes intramolecular cascade radical cyclizations of cyanamide radical precursors conveniently to afford polycyclic N-heterocycles in moderate to good yields.
\end{abstract}

Keywords: Cascade radical cyclizations, samarium diiodide, cyanamides, N-heterocycles

\section{Introduction}

Over the past several years, cascade radical cyclizations, in which the cyclized intermediate directly undergoes further cyclization, have received considerable synthetic attention because they have shown great utility as highly effective methods to construct polycyclic structures in one step..$^{1-4}$ Recently, some natural products have been efficiently synthesized on the basis of cascade radical cyclizations..$^{5-7}$

Samarium diiodide $\left(\mathrm{SmI}_{2}\right)$ has become an exceedingly powerful single electron transfer reagent for promoting reduction and has been applied to a multitude of important synthetic reactions, such as Barbier couplings, Reformatsky reactions, ketone-olefin reductive couplings and pinacol couplings, among which the $\mathrm{SmI}_{2}$-promoted intramolecular cyclizations are particularly attractive. $^{8-13}$

Recently, $N$-acylcyanamides were introduced as radical partners in tributyltin hydride mediated radical cascade cyclizations leading to polycyclic nitrogen heterocycles. ${ }^{14} \mathrm{We}$ report here that $\mathrm{SmI}_{2}$ can promote the radical cascade cyclizations of cyanamide precursors yielding polycyclic $N$ heterocycles.

\section{Results and Discussion}

Scheme 1 shows the synthesis of cyanamides 4 . Condensation of 2 -iodoaniline 1 with dimethyl cyanoimidodithiocarbonate ${ }^{15}$ in the presence of $\mathrm{Cs}_{2} \mathrm{CO}_{3}$ in $\mathrm{DMF}$ at $100{ }^{\circ} \mathrm{C}$ gave adduct 2 . Raney- 
nickel desulfurization of $\mathbf{2}$ in ethanol under reflux afforded 3. Starting from $\mathbf{3}$, cyanamides $\mathbf{4}$ were prepared by two different synthetic routes. Acylation of $\mathbf{3}$ in the presence of $\mathrm{Et}_{3} \mathrm{~N}$ in $\mathrm{CH}_{2} \mathrm{Cl}_{2}$ afforded 4a-d. Substitution at the NH group of compound $\mathbf{3}$ was performed by reaction with alkyl bromides under basic conditions to yield $\mathbf{4 e - g}$.

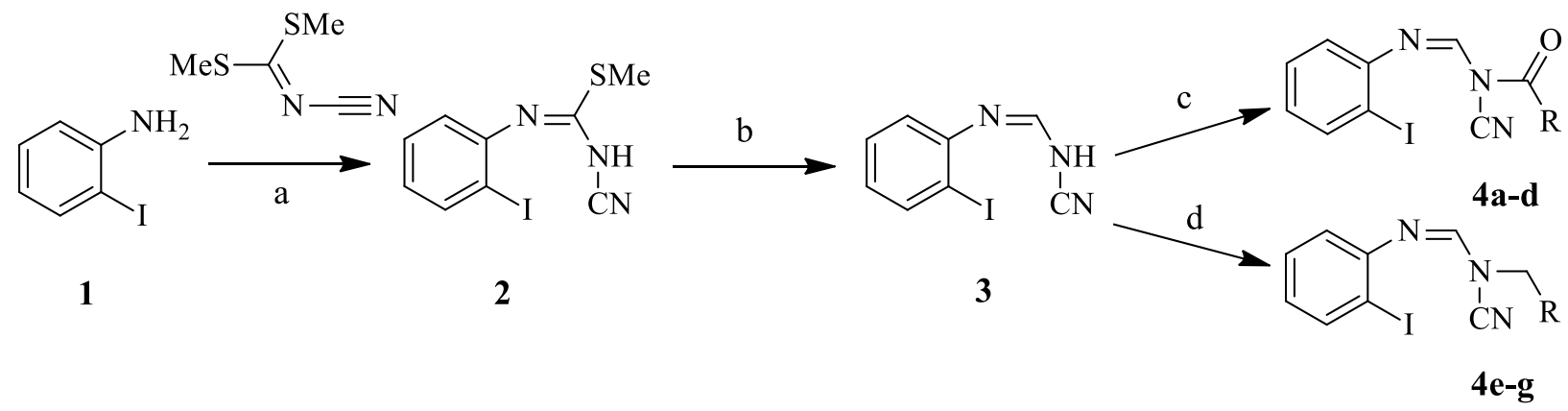

Reagents and conditions: (a) $\mathrm{Cs}_{2} \mathrm{CO}_{3}, \mathrm{DMF}, 100^{\circ} \mathrm{C} ; 41 \%$; (b) Raney Ni, ethanol, reflux; 73\%; (c) $\mathrm{RCOCl}, \mathrm{Et}_{3} \mathrm{~N}, \mathrm{CH}_{2} \mathrm{Cl}_{2} ; 52-71 \%$; (d) $\mathrm{RCH}_{2} \mathrm{Br}, \mathrm{Cs}_{2} \mathrm{CO}_{3}, \mathrm{DMF} ; 42-55 \%$

Scheme 1. The synthetic routes to the cyanamides 4 .

The cyano group is usually stable and cannot be reduced under radical conditions. Occasionally, cyano group shifts can be observed with nitriles. ${ }^{16-18}$ However, substrates $\mathbf{4}$ containing cyano groups formed the intermediate radicals and cyclized to afford $\mathbf{7}$ in moderate to good yields under radical conditions with $\mathrm{SmI}_{2}$ (Scheme 2). The results are listed in Table 1.

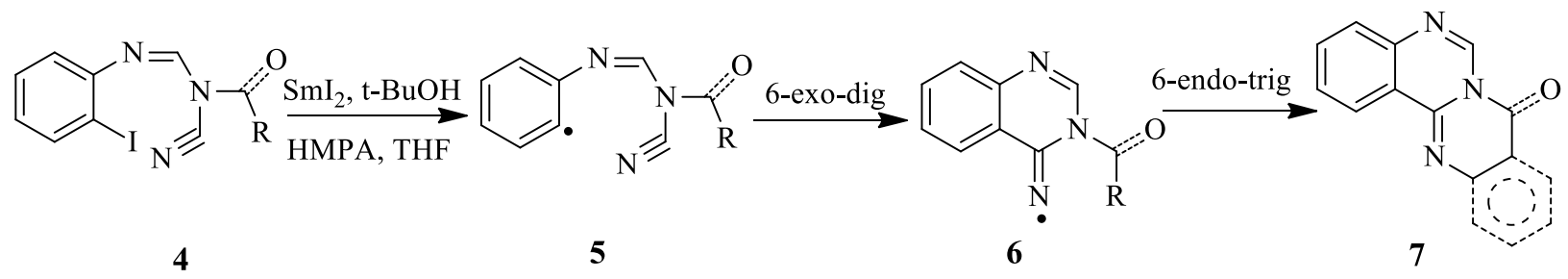

Scheme 2. The intramolecular cascade radical cyclizations promoted by $\mathrm{SmI}_{2}$.

The polycyclic formation can be explained by the putative mechanism presented in Scheme 2 . The aryl radicals 5 generated from the precursors $\mathbf{4}$ underwent a 6-exo-dig cyclization into the nitriles to give the intermediate radicals $\mathbf{6}$. The intermediates $\mathbf{6}$ further underwent a 6-endo-trig addition onto the unsaturated moiety and subsequent aromatization or reduction under the reaction conditions ( $\mathrm{SmI}_{2}-\mathrm{HMPA}$ ) to yield the corresponding polycyclic compounds 7.

The $\mathrm{SmI}_{2}$-mediated reduction of aryl iodide results in a carbon radical which is intramolecularly trapped by a cyanamide, which in turn is trapped by an alkene or a phenyl ring. This methodology is useful in building polycyclic systems in a single step. Moreover, this new process has some methodological advantages since samarium salts are easily eliminated from products. 
Table 1. The heterocyclic compounds 7 prepared by intramolecular cascade cyclizations with $\mathrm{SmI}_{2}$

Entry Precursor

asolated yield.

\section{Conclusions}

In summary, polycyclic $N$-heterocycles can be conveniently prepared via $\mathrm{SmI}_{2}$-promoted intramolecular cascade radical cyclizations with cyanamides as radical precursors. 


\section{Experimental Section}

General. Unless otherwise indicated, all reactions were carried out under a dry nitrogen atmosphere. DMF was freshly distilled from calcium hydride and THF was distilled from sodiumbenzophenone immediately prior to use. The other reagents were used directly without further purification. Melting points (mp) were obtained on a B-540 Büchi melting-point apparatus and are uncorrected. ${ }^{1} \mathrm{H}$ NMR $(400 \mathrm{MHz})$ and ${ }^{13} \mathrm{C}$ NMR $(100 \mathrm{MHz})$ data were recorded on a DPX-400 instrument with $\mathrm{CDCl}_{3}$ or DMSO- $d_{6}$ as solvent and tetramethylsilane (TMS) as the internal standard. Chemical shifts are given in ppm and spin-spin coupling constants, $J$, are given in $\mathrm{Hz}$. Mass spectra (MS) were recorded on a HP5989A mass spectrometer. Elemental analyses were carried out on a PE EA2400 CHN analyzer.

Synthesis of $N$-(2-iodophenyl)- $N$ '-cyano-S-methylisothiourea (2). Dimethyl cyanodithioimidocarbonate $(1.46 \mathrm{~g}, 10 \mathrm{mmol})$ was dissolved in DMF $(20 \mathrm{~mL})$. To the stirred solution were added 2-iodoaniline $(2.19 \mathrm{~g}, 10 \mathrm{mmol})$ and $\mathrm{K}_{2} \mathrm{CO}_{3}(2.07 \mathrm{~g}, 15 \mathrm{mmol})$. The solution was kept at $100{ }^{\circ} \mathrm{C}$ for $8 \mathrm{~h}$ and then poured into ice water. A yellow precipitate formed immediately and was collected by filtration (0.32 g, 41\%). Mp 178-180 ${ }^{\circ} \mathrm{C} ;{ }^{1} \mathrm{H}$ NMR (DMSO- $\left.d_{6}\right) \delta: 10.35$ (s, $\left.1 \mathrm{H}\right), 7.94(\mathrm{~d}, J=8.0 \mathrm{~Hz}, 1 \mathrm{H}$ ), 7.36-7.48 (m, 2H), $7.14\left(\mathrm{t}, J_{1}=7.2 \mathrm{~Hz}, J_{2}=7.2 \mathrm{~Hz}, 1 \mathrm{H}\right), 2.63(\mathrm{~s}, 3 \mathrm{H}) ;{ }^{13} \mathrm{C}$ NMR (DMSO- $\left.d_{6}\right) \delta$ : 161.3, 158.1, 139.2, 130.3, 128.5, 124.1, 116.1, 83.6, 13.3. ESI-MS: $[\mathrm{M}+1]^{+} \mathrm{m} / \mathrm{z}$ 318. Anal. calcd. for $\mathrm{C}_{9} \mathrm{H}_{8} \mathrm{IN}_{3} \mathrm{~S}: \mathrm{C}, 34.08 ; \mathrm{H}, 2.54 ; \mathrm{N}, 13.25$. Found: $\mathrm{C}, 34.17 ; \mathrm{H}, 2.52 ; \mathrm{N}, 13.23$.

Synthesis of $N$-cyano- $N^{\prime}$-(2-iodophenyl)formamidine (3). To a solution of compound 2 (1.2 g, 3.8 $\mathrm{mmol})$ in absolute ethanol $(25 \mathrm{~mL})$ was added Raney Nickel $2800(1.2 \mathrm{~g})$. The mixture was heated to reflux for $6 \mathrm{~h}$ and quickly filtered while it was still hot. The filtrate was concentrated to half its volume and the product was crystallized. The solid was filtered and dried in a vacuum container to afford $3(0.75 \mathrm{~g}, 73 \%)$ as a white solid. Mp $166-168{ }^{\circ} \mathrm{C} ;{ }^{1} \mathrm{H} \mathrm{NMR}\left(\mathrm{CDCl}_{3}\right) \delta: 9.16(\mathrm{~s}, 1 \mathrm{H}), 7.64(\mathrm{~d}, J$ $=7.2 \mathrm{~Hz}, 1 \mathrm{H}), 7.37(\mathrm{~s}, 1 \mathrm{H}), 7.06-7.21(\mathrm{~m}, 2 \mathrm{H}), 6.93\left(\mathrm{t}, J_{l}=7.2 \mathrm{~Hz}, J_{2}=7.2 \mathrm{~Hz}, 1 \mathrm{H}\right) ;{ }^{13} \mathrm{C} \mathrm{NMR}$ $\left(\mathrm{CDCl}_{3}\right) \delta: 160.9,157.3,139.0,129.1,122.7,118.6,115.7,84.2$. ESI-MS: $[\mathrm{M}+1]^{+} \mathrm{m} / \mathrm{z} 272$. Anal. calcd. for $\mathrm{C}_{8} \mathrm{H}_{6} \mathrm{IN}_{3}: \mathrm{C}, 35.45 ; \mathrm{H}, 2.23 ; \mathrm{N}, 15.50$. Found: $\mathrm{C}, 35.37 ; \mathrm{H}, 2.25 ; \mathrm{N}, 15.58$.

\section{General procedure for synthesis of cyanamides (4a-d)}

To a solution of compound $3(0.54 \mathrm{~g}, 0.2 \mathrm{mmol})$ in $\mathrm{CH}_{2} \mathrm{Cl}_{2}(10 \mathrm{~mL})$ was added $\mathrm{Et}_{3} \mathrm{~N}(0.4 \mathrm{mmol})$. The appropriate acyl chloride $(0.3 \mathrm{mmol})$ in $\mathrm{CH}_{2} \mathrm{Cl}_{2}(3 \mathrm{~mL})$ was added dropwise to the mixture at 0 ${ }^{\circ} \mathrm{C}$. The mixture was stirred for $1 \mathrm{~h}$ at room temperature. The reaction was quenched with saturated $\mathrm{NaHCO}_{3}$ solution and then extracted with $\mathrm{CH}_{2} \mathrm{Cl}_{2}$. The organic layer was washed with water, brine and dried over $\mathrm{Na}_{2} \mathrm{SO}_{4}$. The filtrate was evaporated to dryness under reduced pressure, and the residue was purified by silica gel flash chromatography to give 4a-d.

$\mathrm{N}$-Cyano- $\mathrm{N}$-[(2-iodophenylimino)methyl]benzamide (4a). White solid, yield $71 \% ;{ }^{1} \mathrm{H}$ NMR $\left(\mathrm{CDCl}_{3}\right) \delta: 8.13(\mathrm{~s}, 1 \mathrm{H}), 7.61-7.82(\mathrm{~m}, 6 \mathrm{H}), 7.11-7.42(\mathrm{~m}, 3 \mathrm{H}) ;{ }^{13} \mathrm{C} \mathrm{NMR}\left(\mathrm{CDCl}_{3}\right) \delta: 169.6,161.5$, 158.3, 139.2, 135.3, 133.1, 130.1, 129.3, 128.7, 128.1, 124.3, 116.2, 83.6. ESI-MS: $[\mathrm{M}+1]^{+} \mathrm{m} / \mathrm{z}$ 376. Anal. calcd. for $\mathrm{C}_{15} \mathrm{H}_{10} \mathrm{IN}_{3} \mathrm{O}$ : C, 48.02; H, 2.69; N, 11.20. Found: C, 48.08; H, 2.67; N, 11.23. 
$N$-Cyano- $N$-[(2-iodophenylimino)methyl]-4-methylbenzamide (4b). Off-white solid, yield 52\%; ${ }^{1} \mathrm{H} \mathrm{NMR}\left(\mathrm{CDCl}_{3}\right) \delta: 8.09(\mathrm{~s}, 1 \mathrm{H}), 7.12-7.81(\mathrm{~m}, 8 \mathrm{H}), 2.38(\mathrm{~s}, 3 \mathrm{H}) ;{ }^{13} \mathrm{C} \mathrm{NMR}\left(\mathrm{CDCl}_{3}\right) \delta: 169.4$, 161.5, 158.3, 142.0, 139.2, 131.8, 130.1, 129.8, 128.7, 128.0, 124.3, 116.2, 83.7, 24.2. ESI-MS: $[\mathrm{M}+1]^{+} \mathrm{m} / \mathrm{z}$ 390. Anal. calcd. for $\mathrm{C}_{16} \mathrm{H}_{12} \mathrm{IN}_{3} \mathrm{O}: \mathrm{C}, 49.38 ; \mathrm{H}, 3.11 ; \mathrm{N}, 10.80$. Found: C, 49.45; H, $3.14 ; \mathrm{N}, 10.85$.

$N$-Cyano- $N$-[(2-iodophenylimino)methyl]-3-methylbenzamide (4c). Off-white solid, yield 64\%; ${ }^{1} \mathrm{H} \mathrm{NMR}\left(\mathrm{CDCl}_{3}\right) \delta: 8.10(\mathrm{~s}, 1 \mathrm{H}), 7.13-7.79(\mathrm{~m}, 8 \mathrm{H}), 2.36(\mathrm{~s}, 3 \mathrm{H}) ;{ }^{13} \mathrm{C} \mathrm{NMR}\left(\mathrm{CDCl}_{3}\right) \delta: 169.5$, 161.5, 158.2, 139.1, 138.7, 133.9, 132.2, 129.6, 129.1, 128.8, 128.5, 124.7, 124.3, 116.1, 83.6, 24.0. ESI-MS: $[\mathrm{M}+1]^{+} \mathrm{m} / \mathrm{z}$ 390. Anal. calcd. for $\mathrm{C}_{16} \mathrm{H}_{12} \mathrm{IN}_{3} \mathrm{O}: \mathrm{C}, 49.38 ; \mathrm{H}, 3.11 ; \mathrm{N}, 10.80$. Found: C, 49.45; H, 3.13; N, 10.85.

3-Bromo- $N$-cyano- $N$-[(2-iodophenylimino)methyl]benzamide (4d). Pale white solid, yield 58\%; ${ }^{1} \mathrm{H}$ NMR $\left(\mathrm{CDCl}_{3}\right) \delta: 8.25(\mathrm{~s}, 1 \mathrm{H}), 7.47-7.93(\mathrm{~m}, 6 \mathrm{H}), 7.12-7.36(\mathrm{~m}, 2 \mathrm{H}) ;{ }^{13} \mathrm{C} \mathrm{NMR}\left(\mathrm{CDCl}_{3}\right) \delta$ : 169.6, 161.6, 158.2, 139.1, 136.2, 134.9, 131.1, 129.6, 129.4, 128.8, 126.2, 124.3, 123.5, 116.3, 83.6. ESI-MS: $[\mathrm{M}+1]^{+} \mathrm{m} / \mathrm{z}$ 455. Anal. calcd. for $\mathrm{C}_{15} \mathrm{H}_{9} \mathrm{BrIN}_{3} \mathrm{O}$ : C, 39.68; H, 2.00; N, 9.25. Found: C, 39.62; H, 2.01; N, 9.15.

\section{General procedure for synthesis of cyanamides $(4 \mathrm{e}-\mathrm{g})$}

To a solution of compound $3(0.54 \mathrm{~g}, 0.2 \mathrm{mmol})$ in DMF $(10 \mathrm{~mL})$ was added $\mathrm{Cs}_{2} \mathrm{CO}_{3}(0.4 \mathrm{mmol})$. Alkyl bromide $(0.22 \mathrm{mmol})$ was added dropwise to the mixture. The mixture was stirred for $8 \mathrm{~h}$ at $50{ }^{\circ} \mathrm{C}$. After filtration, the filtrate was concentrated and the residue was purified by silica gel flash chromatography to give $\mathbf{4 e - g}$.

$\boldsymbol{N}$-Benzyl- $\boldsymbol{N}$-cyano- $\boldsymbol{N}$ '-(2-iodophenyl)formamidine (4e). White solid, yield $55 \%$; ${ }^{1} \mathrm{H}$ NMR $\left(\mathrm{CDCl}_{3}\right) \delta$ : $7.91(\mathrm{~s}, 1 \mathrm{H}), 7.08-7.83(\mathrm{~m}, 9 \mathrm{H}), 4.11(\mathrm{~s}, 2 \mathrm{H}) ;{ }^{13} \mathrm{C} \mathrm{NMR}\left(\mathrm{CDCl}_{3}\right) \delta: 161.1,158.2,141.2$, 139.2, 130.1, 129.3, 128.7, 127.2, 126.5, 124.3, 116.1, 83.7, 53.2. ESI-MS: $[\mathrm{M}+1]^{+} \mathrm{m} / \mathrm{z}$ 362. Anal. calcd. for $\mathrm{C}_{15} \mathrm{H}_{12} \mathrm{IN}_{3}$ : C, 49.88; H, 3.35; N, 11.63. Found: C, 49.81; H, 3.28; N, 11.35.

$\boldsymbol{N}$-Cyano- $\boldsymbol{N}^{\prime}$-(2-iodophenyl)- $\boldsymbol{N}$-(3-methylbenzyl)formamidine (4f). White solid, yield $47 \%$; ${ }^{1} \mathrm{H}$ NMR $\left(\mathrm{CDCl}_{3}\right) \delta: 7.91(\mathrm{~s}, 1 \mathrm{H}), 7.11-7.84(\mathrm{~m}, 8 \mathrm{H}), 4.11(\mathrm{~s}, 2 \mathrm{H}), 2.38(\mathrm{~s}, 3 \mathrm{H}) ;{ }^{13} \mathrm{C} \mathrm{NMR}\left(\mathrm{CDCl}_{3}\right) \delta$ : 161.1, 158.2, 141.1, 139.1, 138.4, 130.1, 129.2, 128.8, 128.5, 127.2, 124.4, 124.3, 116.1, 83.6, 53.3, 24.4. ESI-MS: $[\mathrm{M}+1]^{+} \mathrm{m} / \mathrm{z}$ 376. Anal. calcd. for $\mathrm{C}_{16} \mathrm{H}_{14} \mathrm{IN}_{3}$ : C, 51.22; $\mathrm{H}, 3.76 ; \mathrm{N}, 11.20$. Found: $\mathrm{C}$, $51.13 ; \mathrm{H}, 3.73 ; \mathrm{N}, 11.15$.

$\boldsymbol{N}$-Allyl- $\boldsymbol{N}$-cyano- $\boldsymbol{N}$ '-(2-iodophenyl)formamidine (4g). White solid, yield $42 \% ;{ }^{1} \mathrm{H} \mathrm{NMR}\left(\mathrm{CDCl}_{3}\right)$ $\delta: 7.91(\mathrm{~s}, 1 \mathrm{H}), 7.31-7.84(\mathrm{~m}, 4 \mathrm{H}), 5.76(\mathrm{~m}, 1 \mathrm{H}), 5.18\left(\mathrm{dd}, J_{l}=16.4 \mathrm{~Hz}, J_{2}=10.4 \mathrm{~Hz}, 1 \mathrm{H}\right), 5.03(\mathrm{dd}$, $\left.J_{l}=10.4 \mathrm{~Hz}, J_{2}=1.2 \mathrm{~Hz}, 1 \mathrm{H}\right), 3.38-3.44(\mathrm{~m}, 2 \mathrm{H}) ;{ }^{13} \mathrm{C} \mathrm{NMR}\left(\mathrm{CDCl}_{3}\right) \delta: 161.0,158.1,139.1,135.1$, 129.3, 128.8, 124.3, 116.7, 116.2, 83.7, 50.3. ESI-MS: $[\mathrm{M}+1]^{+} \mathrm{m} / \mathrm{z}$ 312. Anal. calcd. for $\mathrm{C}_{11} \mathrm{H}_{10} \mathrm{IN}_{3}$ : C, 42.46; H, 3.24; N, 13.51. Found: C, 42.52; H, 3.22; N, 13.48 .

\section{Typical procedure for the $\mathrm{SmI}_{2}$-promoted cyclization reactions}

A solution of $t$-butanol $(155 \mathrm{mg}, 2.1 \mathrm{mmol})$ in THF $(1 \mathrm{~mL})$ and cyanamide $4(0.7 \mathrm{mmol})$ were added to a solution of HMPA $(1.75 \mathrm{~g}, 10.0 \mathrm{mmol})$ and $\mathrm{SmI}_{2}(0.1 \mathrm{M}$ in THF, $21 \mathrm{~mL}, 2.1 \mathrm{mmol})$ at room temperature. The reaction mixture was subjected to TLC analysis until the reaction was completed. The reaction was quenched with $0.1 \mathrm{M} \mathrm{HCl}$ and extracted with ether. The ether layer 
was washed with saturated $\mathrm{NaHCO}_{3}$, water and brine. The organic phase was dried over $\mathrm{Na}_{2} \mathrm{SO}_{4}$ and concentrated under reduced pressure. Purification of the residue by silica gel flash chromatography afforded the cyclization products 7 .

8H-Quinazolino[4,3-b]quinazolin-8-one (7a). Yellow white solid; mp 194-196 ${ }^{\circ} \mathrm{C}$ (lit. ${ }^{19} 197{ }^{\circ} \mathrm{C}$; lit. ${ }^{20} 198{ }^{\circ} \mathrm{C}$ ); ${ }^{1} \mathrm{H}$ NMR (DMSO-d 6$) \delta: 9.33(\mathrm{~s}, 1 \mathrm{H}), 8.86(\mathrm{~d}, J=7.8 \mathrm{~Hz}, 1 \mathrm{H}), 7.59-8.71(\mathrm{~m}, 6 \mathrm{H}), 7.52$ $(\mathrm{m}, 1 \mathrm{H}) ;{ }^{13} \mathrm{C}$ NMR (DMSO-d $) \delta: 160.2,148.4,145.5,144.3,138.4,137.1,134.2,130.0,129.2$, 128.6, 128.3, 128.0, 126.4, 122.7, 119.1. ESI-MS: $[\mathrm{M}+1]^{+} \mathrm{m} / \mathrm{z} 248$.

11-Methyl-8H-quinazolino[4,3-b]quinazolin-8-one (7b). Yellow white solid; mp $186-188{ }^{\circ} \mathrm{C} ;{ }^{1} \mathrm{H}$ NMR (DMSO- $\left.d_{6}\right) \delta: 9.35(\mathrm{~s}, 1 \mathrm{H}), 8.76(\mathrm{~d}, J=7.8 \mathrm{~Hz}, 1 \mathrm{H}), 7.47-7.89(\mathrm{~m}, 6 \mathrm{H}), 2.48(\mathrm{~s}, 3 \mathrm{H}) ;{ }^{13} \mathrm{C}$ NMR (DMSO- $\left.d_{6}\right) \delta: 160.1,148.4,145.4,144.3,140.5,138.2,134.2,130.1,128.9,128.6,128.3$, 128.0, 127.7, 121.4, 119.0, 21.3. HRMS m/z calcd. for $\mathrm{C}_{16} \mathrm{H}_{11} \mathrm{~N}_{3} \mathrm{O}\left(\mathrm{M}^{+}\right)$261.0902, found 261.0909. Anal. calcd. for $\mathrm{C}_{16} \mathrm{H}_{11} \mathrm{~N}_{3} \mathrm{O}: \mathrm{C}, 73.55 ; \mathrm{H}, 4.24 ; \mathrm{N}, 16.08$. Found: C, 73.32; H, 4.12; N, 15.93 .

10-Methyl-8H-quinazolino[4,3-b]quinazolin-8-one (7c). Yellow white solid; mp 201-203 ${ }^{\circ} \mathrm{C}$ (lit. $\left.{ }^{20} 211{ }^{\circ} \mathrm{C}\right) ;{ }^{1} \mathrm{H}$ NMR (DMSO-d6) $\delta: 9.35(\mathrm{~s}, 1 \mathrm{H}), 8.78(\mathrm{~d}, J=7.8 \mathrm{~Hz}, 1 \mathrm{H}), 8.36(\mathrm{~s}, 1 \mathrm{H}), 7.55-7.93(\mathrm{~m}$, $5 \mathrm{H}), 2.48(\mathrm{~s}, 3 \mathrm{H}) ;{ }^{13} \mathrm{C}$ NMR (DMSO-d6) $\delta: 160.2,148.3,145.5,141.6,138.4,137.0,136.8,134.2$, 130.2, 129.2, 128.6, 128.1, 126.4, 122.6, 119.0, 21.4. ESI-MS: $[\mathrm{M}+1]^{+} \mathrm{m} / \mathrm{z} 262$.

10-Bromo-8H-quinazolino[4,3-b]quinazolin-8-one (7d). Yellow solid; mp 211-212 ${ }^{\circ} \mathrm{C}$ (lit. ${ }^{20} 229$ $\left.{ }^{\circ} \mathrm{C}\right) ;{ }^{1} \mathrm{H}$ NMR (DMSO-d6) $\delta: 9.36(\mathrm{~s}, 1 \mathrm{H}), 8.77(\mathrm{~d}, J=8.0 \mathrm{~Hz}, 1 \mathrm{H}), 8.51(\mathrm{~s}, 1 \mathrm{H}), 7.64-8.07(\mathrm{~m}, 5 \mathrm{H})$; ${ }^{13} \mathrm{C}$ NMR (DMSO- $\left.d_{6}\right) \delta$ : 160.2, 148.4, 145.5, 143.8, 138.9, 138.4, 134.2, 133.1, 130.0, 129.2, 128.6, 126.4, 123.7, 124.4, 119.0. ESI-MS: $[\mathrm{M}+1]^{+} \mathrm{m} / \mathrm{z} 326$.

8H-Quinazolino[4,3-b]quinazoline (7e). Yellow solid; mp 167-169 ${ }^{\circ} \mathrm{C} ;{ }^{1} \mathrm{H}$ NMR (DMSO- $d_{6}$, $\delta$ :

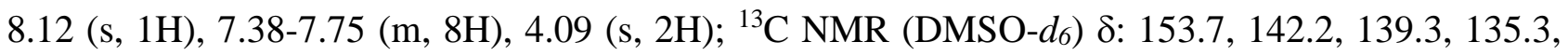
134.2, 132.6, 130.0, 129.2, 128.6, 128.3, 128.0, 126.3, 125.8, 119.1, 49.7. HRMS m/z calcd. for $\mathrm{C}_{15} \mathrm{H}_{11} \mathrm{~N}_{3}\left(\mathrm{M}^{+}\right)$233.0953, found 233.0958. Anal. calcd. for $\mathrm{C}_{15} \mathrm{H}_{11} \mathrm{~N}_{3}$ : C, 77.23; H, 4.75; N, 18.01. Found: C, 77.14; H, 4.71; N, 17.86 .

10-Methyl-8H-quinazolino[4,3-b]quinazoline (7f). Yellow solid; mp 154-156 ${ }^{\circ} \mathrm{C} ;{ }^{1} \mathrm{H}$ NMR $\left(\mathrm{DMSO}-d_{6}\right) \delta: 8.12(\mathrm{~s}, 1 \mathrm{H}), 7.11-7.73(\mathrm{~m}, 7 \mathrm{H}), 4.09(\mathrm{~s}, 2 \mathrm{H}), 2.37(\mathrm{~s}, 3 \mathrm{H}) ;{ }^{13} \mathrm{C} \mathrm{NMR}\left(\mathrm{DMSO}-d_{6}\right) \delta$ : 153.7, 140.4, 139.5, 136.7, 135.3, 134.2, 132.6, 131.8, 129.1, 128.6, 128.0, 126.2, 125.8, 119.0, 49.9, 21.8. HRMS m/z calcd. for $\mathrm{C}_{16} \mathrm{H}_{13} \mathrm{~N}_{3}\left(\mathrm{M}^{+}\right)$247.1109, found 247.1116. Anal. calcd. for $\mathrm{C}_{16} \mathrm{H}_{13} \mathrm{~N}_{3}$ : C, 77.71; H, 5.30; N, 16.99. Found: C, 77.82; H, 5.35; N, 17.13.

3,4-Dihydro-2H-pyrimido[1,2-c]quinazoline (7g). Brown oil; ${ }^{1} \mathrm{H}$ NMR (DMSO-d6) $\delta$ : 7.93(s, 1H), 7.28-7.61(m, 4H), 4.84(t, 2H), 3.62(t, 2H), 1.98(m,2H); ${ }^{13} \mathrm{C}$ NMR (DMSO-d $\left.d_{6}\right) \delta: 142.9,140.6$, 138.3, 131.7, 129.2, 126.2, 123.7, 120.1, 45.3, 37.9, 18.4. HRMS m/z calcd. for $\mathrm{C}_{11} \mathrm{H}_{11} \mathrm{~N}_{3}\left(\mathrm{M}^{+}\right)$ 185.0953, found 185.0961. Anal. calcd. for $\mathrm{C}_{11} \mathrm{H}_{11} \mathrm{~N}_{3}$ : C, 71.33; H, 5.99; N, 22.69. Found: C, 71.51; H, 6.07; N, 22.88.

\section{Acknowledgements}

Zhang $\mathrm{Hu}$ thanks for the Program for Excellent Talents in Guangdong Ocean University (No. 0912118), and thanks Professor Yongping Yu for helpful discussions. 


\section{References}

1. Leca, D.; Fensterbank, L.; Lacôte, E.; Malacria, M. Angew. Chem. Int. Ed. 2004, 43, 4220.

2. Takasu, K.; Ohsato, H.; Kuroyanagi, J.; Ihara, M. J. Org. Chem. 2002, 67, 6001.

3. Blaszykowski, C.; Dhimane, A. L.; Fensterbank, L.; Malacria, M. Org. Lett. 2003, 5, 1341.

4. Marion, F.; Courillon, C.; Malacria, M. Org. Lett. 2003, 5, 5095.

5. Taniguchi, T.; Tanabe, G.; Muraoka, O.; Ishibashi, H. Org. Lett. 2008, 10, 197.

6. Mejía-Oneto, J. M.; Padwa, A. Org. Lett. 2006, 8, 3275.

7. Taniguchi, T.; Iwasaki, K.; Uchiyama, M.; Tamura, O.; Ishibashi, H. Org. Lett. 2005, 7, 4389.

8. Krief, A.; Laval, A. M. Chem. Rev. 1999, 99, 745.

9. Tamiya, H.; Goto, K.; Matsuda, F. Org. Lett. 2004, 6, 545.

10. Kan, T.; Hosokawa, S.; Nara, S.; Tamiya, H.; Shirahama, H.; Matsuda, F. J. Org. Chem. 2004, 69, 8956.

11. Powell, J. R.; Dixon, S.; Light, M. E.; Kilburn, J. D. Tetrahedron Lett. 2009, 50, 3564.

12. Gopalaiah, K.; Kagan, H. B. New J. Chem. 2008, 32, 607.

13. Saadi, J.; Lentz, D.; Reissig, H. U. Org. Lett. 2009, 11, 3334.

14. Servais, A.; Azzouz, M.; Lopes, D.; Courillon, C.; Malacria, M. Angew. Chem. Int. Ed. 2007, $46,576$.

15. Alqaradawi, S. Y.; Elgemeie, G. H. Synth. Commun. 2004, 34, 805.

16. Crich, D.; Bowers, A. A. Org. Lett. 2006, 8, 4327.

17. Crich, D.; Bowers, A. A. J. Org. Chem. 2006, 71, 3452.

18. Beckwith, A. L. J.; O’Shea, D. M.; Westwood, S. W. J. Am. Chem. Soc. 1988, 110, 2565.

19. Plescia, S.; Ajello, E.; Sprio, V.; Marino, M. L. J. Heterocycl. Chem. 1974, 11, 603.

20. Alexandre, F. R.; Berecibar, A.; Wrigglesworth, R.; Besson, T. Tetrahedron 2003, 59, 1413. 